\title{
Médiévales
}

Langues, Textes, Histoire

67 | automne 2014

Histoires de Bohême

Martine HENNARD DUTHEIL DE LA ROCHÈRE et Véronique

DASEN éd., Des fata aux fées : regards croisés de

l'Antiquité à nos jours

Études de Lettres, 289 (2011), 426 p.

\section{Anna Caiozzo}

\section{(2) OpenEdition}

Journals

Édition électronique

URL : https://journals.openedition.org/medievales/7329

DOI : $10.4000 /$ medievales. 7329

ISSN : $1777-5892$

Éditeur

Presses universitaires de Vincennes

Édition imprimée

Date de publication : 31 décembre 2014

Pagination : 189-192

ISBN : 978-2-84292-422-5

ISSN : 0751-2708

Référence électronique

Anna Caiozzo, « Martine hennard dutheIL de LA Rochère et Véronique dasen éd., Des fata aux fées : regards croisés de l'Antiquité à nos jours », Médiévales [En ligne], 67 | automne 2014, mis en ligne le 09 mars 2015, consulté le 23 avril 2022. URL : http://journals.openedition.org/medievales/7329 ; DOI : https:// doi.org/10.4000/medievales.7329

Ce document a été généré automatiquement le 23 avril 2022.

Tous droits réservés 


\section{Martine HENNARD DUTHEIL DE LA ROCHÈRE et Véronique DASEN éd., Des fata aux fées : regards croisés de l'Antiquité à nos jours}

Études de Lettres, 289 (2011), 426 p.

\section{Anna Caiozzo}

\section{RÉFÉRENCE}

Martine HENNARD DUTHEIL DE LA ROCHÈRE et Véronique DASEN éd., Des fata aux fées : regards croisés de l'Antiquité à nos jours, Études de Lettres, 289 (2011), 426 p.

1 Le volume " Des fata aux fées ${ }^{1}$ » est un collectif de dix-huit articles issus d'un colloque tenu à Lausanne en 2009 évoquant la vie des contes de fées, à travers lieux et époques, de l'Antiquité à nos jours. Dans l'introduction, Martine Hennard Dutheil de la Rochère et Véronique Dasen présentent le personnage de la fée, cette accoucheuse des rêves qui "met en lumière l'expérience intime et les ressorts inconscient de la psyché humaine » (p. 15). Elles précisent l'origine du terme fée issu du latin fata, « destin ». Toutefois, on peut mentionner que David Bivar proposait une autre étymologie issue du monde iranien, pairi ou pairaka, qui aurait pu donner " fairy» et donc " fée $»^{2}$; mais, rappelonsle, la fée est aussi un démon d'origine atmosphérique, une entité qui renvoie aux concepts d'équilibre de la nature, et des forces que l'on met en présence pour assurer sa pérennités.

2 Trois thèmes structurent l'ouvrage autour du rôle de la fée: les anciennes représentations des rites de naissance, les liens entre l'héritage gréco-romain et les contes de fées modernes et, enfin, le motif retravaillé de la Belle au bois dormant dans différentes cultures et époques. 
3 Les anciennes représentations des rites de naissance permettent de comprendre comment la parole prophétique de la fée scelle le destin de l'individu dès la naissance, destin inscrit par exemple sur la tablette tenue en Mésopotamie par le père des dieux, Enlil, d'après la passionnante contribution de Constance Frank, dans « Le fuseau et la quenouille ». Personnalités divines et humaines participent à la naissance de l'homme et à sa destinée en Mésopotamie ancienne. L'auteure explique comment l'acte de naître rejoue la création originelle et met en scène les protagonistes de l'acte primordial. Elle analyse ainsi les personnalités divines et humaines qui participent à la naissance, les procédés incantatoires, l'iconographie des rites de naissance, les dangers et entités qui menacent le nouveau-né. Le rôle d'Aruru Nintu, la sage-femme modèle, est particulièrement intéressant; usant du roseau pour couper le cordon, c'est elle qui prononce le bon mot pour fixer la destinée de l'enfant qu'elle protège aussi de la lamashtû et du kûbu, l'avorton. Par ailleurs, Gula/Ninkarrak, déesse de la médecine, intervient comme shabsûtu ou sage-femme; elle agit sur la dimension sexuée et présente à la fille un fuseau et une quenouille et, au garçon, un boomerang et une arme. L'auteure note qu'entre toutes les divinités qui interviennent à la naissance, il se produit de constantes confusions. Pour le monde égyptien, Cathie Spieser évoque la naissance en musique, le destin fixé par Isis, les quatre Meskhenet et les sept Hathor-s qui se retrouvent ailleurs sous d'autres formes. Les déesses sont associées à des bandelettes rouges, une probable évocation du cordon ombilical tenu par Anubis comme promesse de renaissance, couleur du sang et étoffe de Tayt, déesse du tissage. Vinciane Pirenne-Delforge et Gabriela Pironti présentent les Moires du monde grec qui, depuis Homère, président à la naissance et à la mort, incarnant la figure de la fileuse, de la tisserande, et la trame de la vie. Véronique Dasen, dans « Le pouvoir des femmes : des Parques aux Matres", développe le rôle de la sage-femme qui vérifie la viabilité de l'enfant et fait les gestes rituels d'entrée dans la vie, depuis la rupture du cordon jusqu'au premier bain, alors que ce sont les Parques qui, elles, en déterminent la longévité. L'auteure analyse les images de la naissance dans l'art, les gemmes pour repérer le fameux rôle attribué au père qui, de fait, ne participe pas à l'accouchement, contrairement aux idées reçues.

Dans la seconde partie consacrée aux réécritures littéraires,les sources mythiques gréco-romaines alimentent le merveilleux médiéval et les fées apparaissent comme des figures ambivalentes, maternelles et séductrices, ouvrant le débat sur la question du statut de la femme vis-à-vis de la sexualité. Jacqueline Fabre-Serris, dans « L'Histoire de Méléagre vue par Ovide ou de quoi le tison des Parques est-il l'emblème? ", propose une très belle analyse du tison, ce lien évocateurentre l'homme et les femmes de la famille, qui symbolise le fil de la vie. Jean-Claude Mühlethaler, dans «Translittérations féeriques au Moyen Âge de Mélior à Mélusine, entre histoire et fiction »,interroge le féminin sur des récits médiévaux féériques et les sources qui les inspirent: Partenopeu de Blois, par exemple, est inspiré d'Apulée et du folklore celtique mais aussi du roman courtois. Ici, le merveilleux féerique est associé à la découverte de la sexualité. Le statut de la femme au Moyen Âge est parfaitement décrit à travers la figure de certaines fées, telle la Mélusine de Jean d'Arras, épouse et mère, figure tutélaire des Lusignan. La fée révèle le héros à lui-même tout en demeurant un être mystérieux. L'époque moderne fut riche en figures féeriques, liées ou non à l'œuvre de Perrault. Noémie Chardonnens traite, dans « D'un imaginaire à l'autre, la belle endormie du Roman de Perceforest et son fils », des fées marraines, tisserandes fatales ou fées de cour qui inspirèrent les contes de Perrault, en évoquant en filigrane le sort des filles à marier sous l'Ancien Régime, 
comme le montre encore Ute Heidmann dans «Tisserandes fatales (Apulée) et fées de cour (Perrault): le sort difficile d'une belle "née pour être couronnée" ». Sylvie Ballestra-Puech ( $(\mathrm{Du}$ fil des Parques au fil des fées, la fabrique du conte dans "Serpentin vert de Madame d'Aulnoy" ") explicite la réception des Parques dans l'œuvre de Marie-Catherine d'Aulnoy, contemporaine de Perrault, ce qui conduit l'auteure à une réflexion sur le genre, la condition féminine et le rapport amoureux. L'héroïne du Serpentin vert doit filer, à la demande de la fée Margotine, son destin et celui de son amoureux, dans un conte qui précise comment le fil du destin conduit à celui de l'écriture. Magali Monnier, dans « Naissance et Renaissance du conte de fées de Marie-Catherine d'Aulnoy à Angela Carter ", évalue l'héritage de Madame d'Aulnoy dans la littérature contemporaine anglaise et la dimension genrée du conte qui évoque la condition des femmes à leur époque respective. Cyrille François («Fées et weise Frauen. Les faiseuses de dons chez Perrault et les Grimm, du merveilleux rationalisé au merveilleux naturalisé ») analyse quant à lui les fées de Perrault dans le contexte des Lumières, et leurs homologues germaniques chez Grimm dans le contexte des identités nationales, une perspective intéressante. Donald Haase («Kiss and Tell: Orality, Narrative, and the Power of Words in "Sleeping Beauty" ») explicite la dimension métafictionnelle du conte à partir de la tension entre parole et écriture: le baiser métaphore de la réception du conte.

5 Une troisième partie illustre la transmission culturelle à travers les âges par le biais de l'illustration et de la littérature de jeunesse, de la photographie, du dessin animé, de la radio et du cinéma. Un des motifs les plus traités fut celui de la Belle endormie et de la fée fatale. Philippe Kaenel met en relief, avec « Féerique et macabre : l'art de Gustave Doré ", la dimension funeste de l'œuvre du célèbre illustrateur, et Michel Viegnes, dans «La force au féminin dans le conte merveilleux de fin de siècle », insiste, lui, sur le conte merveilleux fin-de-siècle chez Théodore de Banville, Catulle Mendès, Henri de Régnier, ou encore Marcel Schwob où les très jeunes femmes détentrices de savoir et de facultés surnaturelles sont les réminiscences d'un monde condamné par la modernité. Martine Hennard Dutheil de la Rochère analyse les œuvres cultes d'Angela Carter, Vampirella et The Lady of the House of Love, des histoires de vampires que l'on peut voir comme des relectures à rebours du conte, forme de transposition dans la culture populaire. Elisabeth Wanning, dans " Old men and Comatose Virgin ", s'interroge sur la politique du genre à l'œuvre dans la réactivation du topos de la Belle endormie dans les adaptations récentes du conte qui insistent sur la fantasmatique masculine des jeunes femmes endormies, qui ne se réveillent pas (Kawabata, Garcia Marquez), ou sans personnalité, manipulées par des patronnes de maison close. Sylvie Ravussin ( $\mathrm{La}$ disparition des fées dans El verdadero final de la Bella Durmiente d'Ana María Matute ») étudie l'adaptation du conte de Perrault dans le contexte de l'Espagne fasciste. Enfin, dans " "Sleeping Beauty" in Chelmno", Martine Hennard Dutheil de la Rochère et Géraldine Viret s'attardent sur la dimension mémorielle ou la transposition des contes dans le contexte de l'Holocauste dans l'œuvre de Yolen.

6 Tous ces articles passionnants, qui explorent la fée marraine avec le regard de l'historien ou du littéraire comparatiste, démontrent combien les rites de la naissance sont fondamentaux dans les sociétés humaines et que, comme tous rites, ils doivent s'inspirer d'un événement mythique, la naissance des dieux, du monde, rappelant le rôle des êtres de l'au-delà, l'universalité de la condition humaine et le rôle central des 
passeurs pour apprendre à franchir les seuils symboliques, sous peine d'être maudit ou figé, telle la Belle au bois dormant.

\section{NOTES}

1. En ligne : http://edl.revues.org/130.

2. A. D. BIVAR, "A Persian Fairyland», dans A. D. BIVARéd., Papers in Honour Professor Mary Boyce, Leuven, 1985 ("Acta Iranica. Encyclopédie permanente des études iraniennes », 2e série, 11), t.1, p. 25-42.

3. On peut voir l'article de M. TIMUS, « Paraître n'est pas être. Les péris selon le mazdéisme », dans A. CAIOzzo, N. ERNOULT éd., Femmes médiatrices et ambivalentes. Mythes et imaginaires, Paris, 2012, p. 49-62. 